\title{
COVID-19 and bronchial asthma: current perspectives
}

\author{
Masayuki Hojo*, Junko Terada-Hirashima, Haruhito Sugiyama \\ Division of Respiratory Medicine, National Center for Global Health and Medicine, Tokyo, Japan.
}

\begin{abstract}
Angiotensin converting enzyme 2 (ACE2) and transmembrane protease serine 2 (TMPRSS2), two receptors on the cell membrane of bronchial epithelial cells, are indispensable for severe acute respiratory syndrome coronavirus 2 (SARS-CoV-2) infection. ACE2 receptor is increased among aged, males, and smokers. As smoking upsurges ACE2 expression, chronic obstructive pulmonary disease (COPD) patients are prone to SARS-CoV-2 infection, and are at a higher risk for severe forms of COVID-19 (coronavirus disease 2019) once infected. The expression of ACE2 and TMPRSS2 in asthma patients is identical (or less common) to that of healthy participants. ACE2 especially, tends to be low in patients with strong atopic factors and in those with poor asthma control. Therefore, it could be speculated that asthma patients are not susceptible to COVID-19. Epidemiologically, asthma patients are less likely to suffer from COVID-19, and the number of hospitalized patients due to exacerbation of asthma in Japan is also clearly reduced during the COVID-19 pandemic; therefore, they are not aggravating factors for COVID-19. Related academic societies in Japan and abroad still lack clear evidence regarding asthma treatment during the COVID-19 pandemic, and recommend that regular treatment including biologics for severe patients be continued.
\end{abstract}

Keywords: coronavirus disease 2019 (COVID-19), ACE2, ICS, TMPRSS2, biologics

\section{Introduction}

In corona viruses such as severe acute respiratory syndrome coronavirus 2 (SARS-CoV-2), the genome RNA is surrounded by an envelope consisting of a lipid bilayer and an outer membrane protein. SARSCoV-2 initiates invasion into human cells after the Spike protein (S protein, in the envelope) binds to the cell membrane receptor, angiotensin converting enzyme 2 (ACE2). The S protein is cleaved into S1 and S2 by a human cell-derived protease. Then $\mathrm{S} 1$ binds to the ACE2 receptor. The other fragment (S2) is cleaved by transmembrane protease serine 2 (TMPRSS2), a serine protease on the surface of human cells, and as a result, membrane fusion proceeds (1). The gene expression of ACE2 and TMPRSS2 differs depending on the type of tissue/cell. ACE2 is widely expressed in the epithelial cells of the lungs, heart, small intestine, kidney, testicle and liver. TMPRSS2 is also widely expressed in the lungs, kidney, testicle and small intestine. Expression of ACE2 and TMPRSS2 in bronchial epithelial cells is essential for SARS-CoV-2 infection (2).

The expression level of ACE2 is influenced by age, sex, and lifestyle. ACE2 on the cell surface increases with age and generally tends to be denser in males than in females. In addition, the expression level of ACE2 is said to increase with exercise and smoking. The kidney has a high expression level of ACE2 and
TMPRSS2. Although there are many reports of acute renal failure during COVID-19 pandemic, there is currently no clear evidence as to whether SARS-CoV-2 directly infects organs outside the respiratory system (such as the kidneys) (3). The expression level of ACE2 correlates with the susceptibility of infection by SARS$\mathrm{CoV}-2$, and is associated with increased morbidity in patients with respiratory diseases related to smoking. In COVID-19, complications such as hypertension, diabetes mellitus, cardiovascular diseases, chronic renal failure, malignancies (especially in those receiving chemotherapy and radiation therapy) and chronic respiratory diseases are at risk of aggravation.

\section{ACE2 receptor and respiratory diseases}

Since smoking significantly increases the expression of ACE2, patients with chronic obstructive pulmonary disease (COPD, caused by smoking in $90 \%$ of the cases in Japan) are also reported to have increased expression of ACE2 in the airway epithelium (from the bronchial region to the alveoli) (4). This is similar in both current and past smokers. Previous reports show that in COPD patients, airflow limitation progresses and the severity increases with the ACE2 expression level. Epidemiologically, COPD patients are known to be susceptible to COVID-19 and once contracted, the infection is known to be more severe, and correlates 
with ACE2 expression levels (5).

Among chronic respiratory diseases, it has been reported that ACE2 expression is increased in idiopathic pulmonary fibrosis (IPF) as well as COPD (6). Among patients with interstitial pulmonary pneumonitis, reports revealed a significantly higher risk of death in IPF patients (especially those with obesity) (7), and is also presumed to be closely related to ACE2 expression. On the contrary, it has been shown that ACE2 expression tends to be low in sarcoidosis (6). In the following paragraph, we focus on the findings of the expression of each receptor in bronchial asthma.

\section{Bronchial asthma and ACE2 andTMPRSS2 receptors}

According to many epidemiological studies in Japan, the smoking rate in asthma patients is $20 \%-40 \%$, which is the same in healthy individuals. It is presumed that smoking is not involved in the expression of these receptors in asthma patients.

Peters et al. investigated the expression of each receptor using induced sputum in 330 asthma patients (about $60 \%$ of whom were severely ill) who participated in Severe Asthma Research Program-3 (SARP-3). The expression of ICAM-1 (intercellular adhesion molecule-1; the target receptor for rhinovirus and most frequent cause of virus-infection induced exacerbation in asthmatics) was significantly increased in asthma patients compared to 79 healthy individuals, whereas no difference is reported in the expression of ACE2 and TMPRSS2 between the asthma patient group and healthy individuals (8). Sub-analysis has shown that ACE2 expression among asthma patients is significantly increased in the elderly, males, and Africans. Furthermore, stratifying the asthma patients based on the amount of ICS (inhaled corticosteroids) used, it was shown that ACE2 and TMPRSS2 expression was significantly lower in high-dose users than in non-users and low/medium-dose users. On the other hands, Jackson et al. examined the nasal epithelium of 318 pediatric asthma patients and reported that ACE2 expression was significantly lower in patients with high IgE levels and strong atopic factors (9). At the same time, it has been shown that ACE2 expression is significantly reduced in both the nasal and airway epithelium after the post-bronchial allergen-challenge in vivo and incubation with interleukin-13 (IL-13) in vitro. In addition, Kimura et al. examined the nasal mucosa of rhinitis patients and reported that ACE2 expression was significantly lower in patients with stronger Th2 inflammation, and poorer control of comorbid asthma (10).

In summary, the expression of ACE2 and TMPRSS2 is not higher in asthma patients than in healthy subjects. Moreover, ACE2 tends to be low in patients with poor asthma control, strong atopic factors, and high-dose ICS use. From these facts, it could be speculated that asthma patients are not particularly susceptible to COVID-19 and do not become severely ill.

\section{COVID-19 and asthma: findings from epidemiological studies}

Bronchial asthma is a chronic respiratory condition of variable severity characterized by reversible airflow obstruction, airway hyper-responsiveness, and inflammation which results in symptoms such as wheezing, breathlessness, and coughing. In Japan, approximately 3 million people are affected by asthma, and among these, $30 \%$ have moderate asthma, while $7 \%$ have severe asthma. The prevalence of asthma has increased, while its mortality has decreased (1.3 per 100,000 patients in 2018) after introduction of antiinflammatory maintenance treatment mainly by ICS (11). The aim of asthma management is symptom control, and prevention of future risks. Thus, it is important to alleviate airway inflammation and prevent exacerbations which may induce airway remodeling and lead to an intractable condition.

In asthma patients, the antiviral immune response is inadequate, i.e. decrease in the capacity of IFN- $\alpha$ production during a viral infection, compared to healthy individuals; thus, the symptoms are generally exacerbated by respiratory virus infection. Rhinovirus and respiratory syncytial virus are the most common viruses that cause exacerbations, but it is also known that human coronaviruses (four types) are among the most common causes of exacerbation, accounting for about $10 \%$ in all (12). Based on this, theoretically, asthma patients are likely to be infected with SARSCoV-2 and become more severely ill. However, there are few reports of exacerbation of asthma for the same family of coronaviruses such as severe acute respiratory syndrome (SARS) and Middle East respiratory syndrome (MERS) (13).

From an integrated analysis of eight observational studies conducted in China, the United States and Mexico, Matsumoto et al. found that patients with COVID-19 had an asthma complication rate of 5.3\%, lower than the average asthma prevalence of $8.0 \%$ in each region and asthma patients were shown to be less susceptible to COVID-19 (14-16). Other cohorts from the Western nations have also reported less asthma complications in COVID-19 patients requiring hospitalization $(17,18)$. Beurnier et al. reported that 37 (4.8\%) of the 768 hospitalized patients had asthma complications, but none experienced exacerbations of asthma itself during hospitalization (19). Among the asthma patients, $84 \%$ had a Body Mass Index (BMI) of 25 or higher, and a $59 \%$ had other comorbidities such as hypertension $(27 \%)$ and diabetes $(19 \%)$. Chhiba et al. used a computer algorithm in the US to search medical records of patients with COVID-19 confirmed by Polymerase Chain Reaction (PCR) and 
Table 1. Numbers and percentages of asthma and COPD comorbidity in patients with COVID-19

\begin{tabular}{|c|c|c|c|c|c|c|}
\hline \multirow{2}{*}{ Region } & \multirow{2}{*}{$\begin{array}{l}\text { No. of COVID-19 } \\
\text { patients }\end{array}$} & \multirow{2}{*}{$\begin{array}{c}\text { Mean or } \\
\text { median age (y) }\end{array}$} & \multicolumn{2}{|c|}{ No. of comorbid patients $(\%)$} & \multirow{2}{*}{$\begin{array}{l}\text { Regional asthma } \\
\text { prevalence }^{* * *}\end{array}$} & \multirow{2}{*}{ Ref. } \\
\hline & & & Asthma & COPD & & \\
\hline Wuhan, China & 140 & 57 & $0(0)$ & $2(1.4)$ & $6.4 \%$ & Wu F, et al. (37) \\
\hline Wuhan, China & 548 & 60 & $5(0.4)$ & $17(3.1)$ & & Li X, et al. (14) \\
\hline Wuhan, China & 1,590 & 49 & $0(0)$ & $24(1.5)$ & & Guan WJ, et al. (38) \\
\hline Georgia, USA & 305 & 60 & $32(10.5)$ & $16(5.2)$ & $7.7 \%$ & Gold JAW, et al. (39) \\
\hline California, USA & 54 & 54 & $3(0.6)$ & $0(0)$ & & Hartmann S, et al. (40) \\
\hline New York, USA & 5,700 & 63 & $513(9)$ & $308(5.4)$ & & Goyal $\mathrm{P}$, et al. (21) \\
\hline New York, USA & 1,651 & 50 & $99(6)$ & $66(4)$ & & Singer AJ, et al. (15) \\
\hline Chicago, USA & 1,526 & 59 & $220(14.4)$ & NA & & Chhiba KD, et al. (20) \\
\hline Strasburg, France & 106 & 64 & $23(21.6)$ & NA & $10.6 \%$ & Grandbastien M, et al. (17) \\
\hline Paris, France & 768 & 54 & $37(4.8)$ & NA & & Beurnier A, et al. (19) \\
\hline Korea & 7,272 & 56 & $686(9.4)$ & NA & $3.9 \%$ & Lee SC, et al. (22) \\
\hline Italy & 2,000 & 61 & $42(2.1)$ & NA & $6.5 \%$ & Caminati $\mathrm{M}$, et al. (23) \\
\hline Total & 21,660 & - & $1,660(7.7)$ & $433(2)$ & $7.5 \%$ & - \\
\hline
\end{tabular}

"The numbers of patients were calculated only if the total numbers of patients and percentages were presented. ${ }^{* * *}$ Regional asthma prevalence data are cited from The Lancet. 2019; 394:407-418; and CDC, 2020, most recent national asthma data, http://www.cdc.gov/asthma/most_recent_ national_asthma_data.htm.

performed a meta-analysis on the association between asthma/ICS use and hospitalization risk (20). Among the 1,526 patients with COVID-19, 220 (14.4\%) had asthma, but were not associated with an increased risk of hospitalization in a model adjusted for age, gender, and comorbidities. At the same time, the results showed that the use of ICS did not increase the risk of hospitalization. From the above, it could be concluded that asthma patients are less likely to get infected with SARS-CoV-2, and even if they do, asthma exacerbation is unlikely to occur (summarized in Table 1).

A retrospective cohort study of patients admitted to two New York centers found no significant difference in asthma complication rates between non-severe COVID-19 patients (12.2\%) and severe cases needing mechanical ventilation (13.1\%) (21). An integrated analysis by Matsumoto et al. reported that the complication rate of COPD and diabetes was higher in severe cases than in non-severe cases of COVID-19, but there was no difference in the complication rate of asthma (16). On the contrary, according to the Korean nationwide retrospective cohort study using a health insurance database, concomitant asthma was not a significant risk factor for respiratory failure or mortality among all COVID-19 patients (odds ratio [OR]: 0.99, $p=0.997$ and OR: 1.06, $p=0.759$ ) after adjusting for age, sex. However, a history of acute exacerbation in the previous year before COVID-19 was a significant risk factor for death among COVID-19 patients with asthma (OR: 2.63, $p=0.043$ ), especially for the elderly and males (22). Although few patients suffer from COVID-19, many who become severely ill reported that they were originally severely ill according to Global Initiative for Asthma (GINA) treatment step $4 / 5$ (23). From these reports, asthma is generally not a severity factor for COVID-19, but due to the limited evidence in critically ill patients, the US CDC (Center for Disease Control) has stated that "Having moderateto-severe asthma might increase risk for severe illness from COVID-19" (24).

There are limited reports on the current situation in Japan. At our hospital (National Center for Global Health and Medicine), 11 (5.5\%) in-patients from March to August 2020 had a history of asthma. Three patients became ill enough to require oxygen administration, but showed immediate improvement, and there were none that showed exacerbation of asthma. Abe et al. compared and examined changes in the number of patients admitted to acute care hospitals in Japan due to asthma from 2017 to 2020 (25). In 2020, the number of hospitalizations until mid-February (when the first COVID-19 deaths were reported) remained unchanged compared to the previous three years, but since then until the end of May (when the government lifted the state of emergency all over Japan) the number of hospitalizations showed a significant $66 \%$ decrease (95\% confidence interval [CI], 0.37-0.55; $p<0.001$ ). The number of asthma hospitalizations during the pandemic continued to decrease, probably not due to the virus characteristics described above but to the increased preventive measures over this period. Individual-level hygiene measures to prevent COVID-19 might reduce exposure to the strong drivers of asthma exacerbations i.e. infection, allergens exposure, and air pollutants. Moreover, preventive behaviors such as quitting smoking, and better adherence to preventive medications (26) are important possible mechanisms for this drop in asthma hospitalizations.

\section{Treatment for asthma during the COVID-19 pandemic: recommendations from guidelines}

It was initially pointed out that ICS (the basis of asthma treatment) has two potential risks: increasing morbidity, 
and aggravating pneumonia and ARDS after infection. On the contrary, when stratifying patients according to ICS usage, it is suggested that there is a possibility of preventive effect as ACE2 and TMPRSS2 are significantly decreased in high-dose users compared with non-users and low/medium dose users (8).

A systematic review by Halpin et al. stated that there is currently no evidence supporting the fact the use of ICS is detrimental or beneficial to the outcome of COVID-19 (27). In an observational study by Schultze et al., the risk of death was examined in the asthma cohort $(n=818,490)$ with preparations containing ICS vs. short acting 32 -agonists, and in the COPD cohort ( $n$ $=148,557$ ) with preparations containing ICS vs. long acting muscarine agonists/long acting muscarine $\beta 2$ agonists (28). The COPD cohort had a significantly increased risk of COVID-19-related death in the ICS group, while the asthma cohort had a higher risk of death with high-dose ICS, but not with low-dose and medium-dose ICS. As a result of sensitivity analyses, it was concluded that ICS had no adverse effects and can be explained by the severity of the disease at birth. We await further evidence regarding the effect of ICS on COVID-19 patients, but encouragingly, there are no clear reports of worsening cases. Thus, we strongly anticipate the continuation of normal treatment.

GINA, the most widely followed guideline worldwide, created a special chapter of interim guidance on asthma management during the COVID-19 pandemic in their 2020 December update (29) and posted the following guidance:

i) Advise patients to continue taking their prescribed asthma medications, particularly ICS. For patients with severe asthma, continue biologic therapy or oral corticosteroids if prescribed.

ii) Make sure that all patients have a written asthma action plan. The action plan tells the patient how to recognize worsening asthma, how to increase their reliever and controller medications, and when to seek medical help. Take a short course of oral corticosteroids when appropriate for severe asthma exacerbations.

iii) Where possible, avoid nebulizers due to the risk of spreading virus. A pressurized metered-dose inhaler via spacer is preferred except for life-threatening exacerbations. Add a mouthpiece or mask to the spacer if required.

iv) Avoid spirometry in patients with confirmed or suspected COVID-19, or if community transmission of COVID-19 is occurring in your region. Follow strict infection control procedures if aerosol-generating procedures such as nebulization, sputum induction, oxygen therapy and non-invasive ventilation are needed.

The Japanese society of Allergology makes almost the same recommendations in this context.

Molecular-targeted therapy with biologics is also an important option in adult patients with severe asthma.
It has been reported that in patients with COVID-19, cytokines such as IL-4 increase (or remain the same), IL-5 remains the same, and IL-13 increases (30). Recently, there have been case reports of severe asthma patients diagnosed with COVID-19 and treated with an anti-IgE antibody (omalizumab) (31), an anti-IL-5 receptor antibody (benralizumab) $(32,33)$, or an anti-IL$4 \alpha$ receptor antibody (dupilmab) (34), but had a course that eventually tested negative without exacerbation of asthma or pneumonia. Based on this, it is not necessary to cease treatment with biologic agents in asthma patients suffering from COVID-19. At our hospital, about $10 \%$ of asthma patients undergoing outpatient treatment are severe cases who use biologic agents, but none have COVID-19. Regarding the use of biologics in patients with severe asthma, The American Academy of Allergy, Asthma \& Immunology (AAAAI) and the World Allergy Organization (WAO) recommended continuation during the COVID-19 pandemic and noted: "There is no evidence that the drug is harmful to COVID-19, and there is concern that asthma control may worsen due to discontinuation" (35).

Regarding ICS, the Japanese Society of Infectious Diseases is leading the observational study of the administration of ciclesonide that has been found to have an antiviral effect on SARS-CoV-2. At the same time, a multicenter, open-label, randomized trial, led by the National Center for Global Health and Medicine, is underway to investigate its efficacy and safety in treating asymptomatic and mildly ill COVID-19 in patients (36).

In conclusion, it could be speculated that asthma patients are not only unsusceptible to COVID-19, but also they are not aggravating factors for COVID-19, whereas having moderate-to-severe asthma might increase risk for severe illness from COVID-19. Because clear evidence concerning asthma treatment during the COVID-19 pandemic is still lacking, regular long-term treatment such as ICS and biologics for severe patients should be continued.

Funding: This study was supported by Health and Labor Sciences Research Grants (19HA1003).

Conflict of Interest: $\mathrm{MH}$ has, within the last 24 months, received honoraria from AstraZeneca, Glaxo SmithKline, Novartis Pharma, and Boeringer Ingelheim for lectures. Other co-authors have no conflicts of interest to disclose.

\section{References}

1. Clerkin K, Fried JA, Raikhelkar J, Sayer G, Griffin JM, Masoumi A, Jain SS, Burkhoff D, Kumaraiah D, Rabbani L, Schwartz A, Uriel N. COVID-19 and Cardiovascular Disease. Circulation. 2020; 141:1648-1655.

2. Hoffmann M, Kleine-Weber H, Schroeder S, Krüger N, Herrler T, Erichsen S, Schiergens TS, Herrler G, Wu NH, Nitsche A, Müller MA, Drosten C, Pöhlmann S. SARS- 
CoV-2 cell entry depends on ACE2 and TMPRSS2 and is blocked by a clinically proven protease inhibitor. Cell. 2020; 181:271-280.e8.

3. Dong M, Zhang J, Ma X, Tan J, Chen L, Liu S, Xin Y, Zhuang. ACE2, TMPRSS2 distribution and extrapulmonary organ injury in patients with COVID-19. L. Biomed Pharmacother. 2020; 131:110678.

4. Leung JM, Yang CX, Tam A, Shaipanich T, Hackett T, Singhera GK, Dorscheid DR and Sin DD. ACE-2 expression in the small airway epithelia of smokers and COPD patients: implications for COVID-19. Eur Respir J. 2020; 55:2000688.

5. Zhao Q, Meng M, Kumar R, Wu Y, Huang J, Lian N, Deng Y, Lin S. The impact of COPD and smoking history on the severity of COVID-19: A systemic review and meta-analysis. J Med Virol. 2020; 92:1915-1921.

6. Smith J, Sausville EL, Girish V, Yuan ML, Vasudevan A, John KM, Sheltzer JM. Cigarette smoke exposure and inflammatory signaling increase the expression of the SARS-CoV-2 receptor ACE2 in the respiratory tract. Dev Cell. 2020; 53:514-529.

7. Drake TM, Docherty AB, Harrison EM, et al. Outcome of hospitalization for COVID-19 in patients with interstitial lung disease: An international multicenter study. Am J Respir Crit Care Med. 2020; 202:1656-1665.

8. Peters MC, Sajuthi S, Deford P, et al. COVID-19related genes in sputum cells in asthma relationship to demographic features and corticosteroids. Am J Respir Care Crit Med. 2020; 202:83-90.

9. Jackson DJ, Busse WW, Bacharier LB, et al. Association of respiratory allergy, asthma, and expression of the SARS-CoV-2 receptor ACE2. J Allergy Clin Immunol. 2020; 146:203-206.e3.

10. Kimura H, Francisco D, Conway M, Martinez FD, Vercelli D, Polverino F, Billheimer D, Kraft M. Type 2 inflammation modulates ACE2 and TMPRSS2 in airway epithelial cells. J Allergy Clin Immunol. 2020; 146:8088.e8.

11. Nakamura Y, Tamaoki J, Nagase H, Yamaguchi M, Horiguchi T, Hozawa S, Ichinose M, Iwanaga T, Kondo R, Nagata M, Yokoyama A, Tohda Y; Japanese Society of Allergology. Japanese guidelines for adult asthma 2020. Allergol Int. 2020; 69:519-548.

12. Zheng XY, Xu YJ, Guan WJ, Lin LF. Regional, age and respiratory-secretion-specific prevalence of respiratory viruses associated with asthma exacerbation: a literature review. Arch Virol. 2018; 163:845-853.

13. Van Bever HP, Chng SY, Goh DY. Childhood severe acute respiratory syndrome, coronavirus infections and asthma. Pediatr Allergy Immunol. 2004; 15:206-209.

14. Li X, Xu S, Yu M, et al. Risk factors for severity and mortality in adult COVID-19 in patients in Wuhan. J Allergy Clin Immunol. 2020; 146:110-118.

15. Singer AJ, Morley EJ, Meyers K, Fernandes R, Rowe AL, Viccellio P, Thode HC, Bracey A, Henry MC. Cohort of four thousand four hundred four persons under investigation for COVID-19 in a New York hospital and predictors of ICU care and ventilation. Ann Emerg Med. 2020; 76:394-404.

16. Matsumoto K, Daito H. Does asthma affect morbidity or severity of COVID-19? J Allergy Clin Immunol 2020; 146: 55-57.

17. Grandbastien M, Piotin A, Godet J, et al. SARS-CoV-2 pneumonia in hospitalized asthmatic patients did not induce severe exacerbation. J Allergy Clin Immunol
Pract. 2020; 8:2600-2607.

18. Garcia-Pachon E, Zamora-Molina L, Soler-Sempere MJ, Baeza-Martinez C, Grau-Delgado J, Canto-Reig V, Ramon-Sanchez A, Padilla-Navas I, Ruiz-Garcia M, Gonzalo-Jimenez N. Asthma prevalence in patients with SARS-CoV-2 infection detected by RT-PCR not requiring hospitalization. Respir Med. 2020; 171:106084.

19. Beurnier A, Jutant EM, Jevnikar M, et al. Characteristics and outcomes of asthmatic patients with COVID-19 pneumonia who require hospitalization. Eur Respir J. 2020; 56:2001875.

20. Chhiba KD, Patel GB, Vu THT, et al. Prevalence and characterization of asthma in hospitalized and nonhospitalized patients with COVID-19. J Allergy Clin Immunol. 2020; 146: 307-314.e4.

21. Goyal P, Choi JJ, Pinheiro LC, et al. Clinical characteristics of Covid-19 in New York City. N Engl J Med. 2020; 382:2372-2374.

22. Lee SC, Son KJ, Han CH, Jung JY, Park SC. Impact of comorbid asthma on severity of coronavirus disease (COVID-19). Sci Rep. 2020; 10:21805.

23. Caminati M, Vultaggio A, Matucci A, et al. Asthma in a large COVID-19 cohort: Prevalence, features, and determinants of COVID-19 disease severity. Respir Med. 2020; 176:106261.

24. CDC statement; Actions you can take based on your medical conditions and other risk factors (Asthma; moderate-to-severe). https://www.cdc.gov/coronavirus/ 2019-ncov/need-extra-precautions/people-with-medicalconditions.html\#asthma (accessed December 21, 2020).

25. Abe K, Miyawaki A, Nakamura M, Ninomiya H, Kobayashi Y. Trends in hospitalizations for asthma during the COVID-19 outbreak in Japan. J Allergy Clin Immunol Pract. 2021; 9:494-496.e1.

26. Kaye L, Theye B, Smeenk I, Gondalia R, Barrett MA, Stempel DA. Changes in medication adherence among patients with asthma and COPD during the COVID-19 pandemic. J Allergy Clin Immunol Pract. 2020; 8:23842385.

27. Halpin DMG, Singh D, Hadfield RM. Inhaled corticosteroids and COVID-19: a systematic review and clinical perspective. Eur Respir J. 2020; 55:2001009.

28. Schultze A, Walker AJ, MacKenna B, et al. Risk of COVID-19-related death among patients with chronic obstructive pulmonary disease or asthma prescribed inhaled corticosteroids: an observational cohort study using the OpenSAFELY platform. Lancet Respir Med. 2020; 8:1106-1120.

29. Global Initiative for Asthma (GINA). Global strategy for asthma management and prevention: NHLBI/WHO Workshop report: National Heart, Lung and Blood Institute. National Institutes of Health, updated $20 \mathrm{Dec}$ 2020. http://www.ginasthma.com (accessed December 24, 2020)

30. Liu S. Zhi Y, Ying S. COVID-19 and asthma: Reflection during the pandemic. Clinic Rev Allerg Immunol. 2020: 59:78-88.

31. Lommatzsch M, Stoll P, Virchow JC. COVID-19 in a patient with severe asthma treated with Omalizumab. Allergy. 2020; 75:2705-2708.

32. Renner A, Marth K, Patocka K, Pohl W. COVID-19 in a severe eosinophilic asthmatic receiving benralizumab - a case study. J Asthma. 2020; 1-3.

33. García-Moguel I, Díaz Campos R, Alonso Charterina S, Fernández Rodríguez C, Fernández Crespo J. 
COVID-19, severe asthma, and biologics. Ann Allergy Asthma Immunol. 2020; 125:357-359.e1.

34. Bhalla A, Mukherjee M, Radford K, Nazy I, Kjarsgaard M, Bowdish DME, Nair P. Dupilumab, severe asthma airway responses, and SARS-CoV-2 serology. Allergy. 2020; 10.1111/all.14534.

35. Vultaggio A, Agache I, Akdis CA, et al. Considerations on biologicals for patients with allergic disease in times of the COVID-19 pandemic: An EAACI statement. Allergy. 2020; 75:2764-2774.

36. Terada-Hirashima J, Suzuki M, Uemura Y, Hojo M, Mikami A, Sugiura W, Ohmagari N, Sugiyama H. Efficacy and safety of inhaled ciclesonide in treating patients with asymptomatic or mild COVID-19 in the RACCO Trial: Protocol for a multicenter, open-label, randomized controlled trial. JMIR Res Protoc. 2020; 9:e23830.

37. Wu F, Zhao S, Yu B, Chen YM, Wang W, et al. A new coronavirus associated with human respiratory disease in China. Nature. 2020; 579:265-269.

38. Guan WJ, Liang WH, Zhao Y, et al. Comorbidity and its impact on 1590 patients with COVID-19 in China: a nationwide analysis. Eur Respir J. 2020; 55:2000547.

39. Gold JAW, Wong KK, Szablewski CM, et al. Characteristics and clinical outcomes of adult patients hospitalized with COVID-19 - Georgia, March 2020. MMWR Morb Mortal Wkly Rep. 2020; 69:545-550.

40. Hartmann S, Rubin Z, Sato H, OYong K, Terashita D, Balter S. Coronavirus 2019 (COVID-19) infections among healthcare workers, Los Angeles County, February - May 2020. Clin Infect Dis. 2020: ciaa1200.

Received December 26, 2020; Revised February 10, 2021; Accepted February 24, 2021.

Released online in J-STAGE as advance publication March 9, 2021.

*Address correspondence to:

Masayuki Hojo, Division of Respiratory Medicine, National Center for Global Health and Medicine, Toyama 1-21-1, Shinjuku, Tokyo 162-8655, Japan.

E-mail:mhojo@hosp.ncgm.go.jp 